\title{
Nonlinear wave guiding in nematic liquid crystals
}

\author{
Jeroen Beeckman ${ }^{a}$, Kristiaan Neyts ${ }^{a}$, Xavier Hutsebaut ${ }^{b}$, Marc Haelterman ${ }^{b}$ \\ ${ }^{a}$ Liquid Crystals \& Photonics group, ELIS Department, Ghent University, \\ Sint-Pietersnieuwstraat 41, 9000 Gent, Belgium \\ ${ }^{b}$ Service d'optique et acoustique, Université libre de Bruxelles CP 194/5, 50 avenue F.D. \\ Roosevelt, 1050 Bruxelles, Belgium
}

\begin{abstract}
Waveguiding in liquid crystals can be achieved by controlling the molecular orientation by means of external fields or by shaping the geometry of the substrates that contain the liquid crystal material. The creation of these waveguides in liquid crystals can also be achieved by using the optical nonlinear properties of the material. For a sufficient optical power (in the order of a few $\mathrm{mW}$ ), the beam can induce its own optical waveguide. This is a selfinduced waveguide and the resulting beam is referred to as a soliton beam. In the last few years, the properties of these soliton beams have been studied thoroughly, revealing some interesting phenomena. In this article, simulations are reported on two common configurations in which solitons have been generated experimentally. The soliton beam, for certain configurations, displays an undulative behavior inside the cell, which may be used for large angle steering of the optical beam.
\end{abstract}

Keywords: Optical waveguiding, nematic liquid crystals, optical nonlinearity, spatial optical solitons

\section{INTRODUCTION}

Optical beams with finite size always diffract when propagating in free space or uniform media. Depending on the ratio between beam size and wavelength, the diffraction will occur over small or large propagation distances. By making the material nonuniform in terms of refractive index, it is possible to create optical waveguides. These waveguides guide the light along a certain path and prevent the beam from spreading. Usually the light is guided in a region with higher refractive index with respect to the surroundings. When the waveguide is single-mode, only one mode is possible and this mode propagates along the waveguide without changes in amplitude profile.

Nematic liquid crystals are very interesting materials because an optical beam will see a different refractive index depending on the orientation of the molecules with respect to the polarization of the beam. The director is a vector that indicates the local average orientation of the molecules. When the beam is linearly polarized parallel to the director, the refractive index, 'seen' by the light is the extraordinary refractive index $n_{\|}$. When the polarization is perpendicular to the director the light 'sees' the ordinary index of refraction $n_{\perp}$. In this view, it is plausible that, by controlling the orientation of the director, these liquid crystals (LCs) can be used to create waveguides. These waveguides can be created by controlling the local surface orientation of the molecules, ${ }^{1}$ but even more important by applying a voltage over the liquid crystal layer by using appropriate electrodes, ${ }^{2-4}$ i.e. by using the electro-optical properties of the liquid crystal. In this way, the refractive index of the waveguide can be changed and hence the properties of the waveguide, which can be exploited for switching the optical signal or for wavelength tuning of the optical signal. ${ }^{5}$

Another way to create waveguides in liquid crystals is to make use of their optical nonlinearity. ${ }^{6}$ When a material exhibits an optical nonlinearity, such that the refractive index increases with increasing optical field, this leads to self-focusing of the beam. This means that a self-induced waveguide is created in the material. When the self-focusing balances exactly the natural diffraction of the beam, a spatial optical soliton is formed. ${ }^{7}$ Another point of view is that one can only speak of a spatial soliton, when the beam is a mode of its own induced waveguide. During the last decade, these spatial solitons have been observed in nematic liquid crystals in numerous configurations and with different optical nonlinearities.

jeroen.beeckman@elis.ugent.be, phone: +32-9-264.89.51, fax: +32-9-264.35.94 
The remainder of this paper is not included as this paper is copyrighted material. If you wish to obtain an electronic version of this paper, please send an email to bib@elis.UGent.be with a request for publication P107.232.pdf. 